\title{
SONOCHEMISTRY (APPLICATIONS OF ULTRASOUND IN CHEMICAL SYNTHESIS AND REACTIONS): A REVIEW PART I BY
}

\author{
Menshawy A. Mohamed ${ }^{a, b}$ \\ FROM \\ ${ }^{\mathrm{a}}$ Department of Pharmaceutical Chemistry, College of Pharmacy, Prince Sattam Bin \\ Abdulaziz University, P.O. Box- 173, Al-Kharj 11942, Saudi Arabia. \\ ${ }^{\mathrm{b}}$ Department of Organic Chemistry, Faculty of Pharmacy, Al-Azhar University, Cairo, \\ Egypt
}

\begin{abstract}
Sonochemistry is a powerful and green approach which is being used to accelerate synthesis of organic compounds, and involves the use of ultrasound technique to promote chemical reactions. Ultrasound is the part of the sonic spectrum, which ranges from about $20 \mathrm{KHz}$ to $10 \mathrm{MHz}$. A large number of organic reactions can be carried out under ultrasonic irradiation in high yields, short reaction times, and mild conditions. Ultrasonic irradiation of liquids causes high energy chemical reactions to occur, often with the emission of light. The origin of sonochemistry and sonoluminescence is acoustic cavitation which is the formation, growth, and implosive collapse of bubbles. The collapse of bubbles caused by cavitation produces intense local heating and high pressures, with very short lifetimes (Hot spots). These hot-spots have an equivalent temperatures of roughly $5,000 \mathrm{~K}$, pressures of about 1,000 atm, and increase reactivity by nearly million-fold using the radical mechanism. A variety of devices have been used for ultrasonic irradiation of solutions.
\end{abstract}

Keywords: Acoustic cavitation, ultrasound, sonoluminescence, name reactions.

\section{Introduction}

Sonochemistry involves the use of ultrasound technique to promote chemical reactions. Sonochemistry is widely used in organic chemistry, offering versatile and facile pathways for a large variety of syntheses. The study of sonochemistry is concerned with understanding the effect of sonic waves and wave properties of sonchemical systems. Ultrasound is the part of the sonic spectrum, which ranges from about $20 \mathrm{KHz}$ to $10 \mathrm{MHz}$ and can be roughly subdivided in three main regions: low frequency high power ultrasound $(20-100 \mathrm{kHz})$, high frequency medium power ultrasound (100 kHz-1 MHz), and high frequency low power ultrasound (1-10 MHz). Thus, a large number of organic reactions can be carried out under ultrasonic irradiation in high yields, short reaction times, and mild conditions (T.J. Mason and D. Peters,2002, T.J. Mason, 2007, K. Jadidi, et al, 2008) Heterocycles and fused heterocycles exhibit useful biological activities and clinical applications (D.J. Brown, et $a l, 1984)$. Sonochemistry is, thus, an alternative method for rapid and facile synthesis of a variety of heterocycles and fused heterocycles. Ultrasonic-assisted organic synthesis (UAOS) is a powerful and green approach which is being used more and more to accelerate synthesis of organic compounds (H. Xu, et al, 2007, K.P. Guzen, et al , 2007) 
Ultrasonic irradiation of liquids causes high energy chemical reactions to occur, often with the emission of light. The origin of sonochemistry and sonoluminescence is acoustic cavitation: the formation, growth, and implosive collapse of bubbles in liquids irradiated with high intensity sound. The collapse of bubbles caused by cavitation produces intense local heating and high pressures, with very short lifetimes. In clouds of cavitating bubbles, these hot-spots (Suslick, K. S., et al, 1986, Flint, E. B. and Suslick, K. S., 1991, McNamara III, et al, 1999 ) have equivalent temperatures of roughly $5,000 \mathrm{~K}$, pressures of about 1,000 atm, and heating and cooling rates above $10^{10} \mathrm{Ks}$ (T.J. Mason and D. Peters,2002). In single bubble cavitation, conditions may be even more extreme. Thus, cavitation can create extra ordinary physical and chemical conditions in otherwise cold liquids.

When liquids that contain solids were irradiated with ultrasound, related phenomena can occur. When cavitation occurs near an extended solid surface, cavity collapse is non-spherical and drives high-speed jets of liquid into the surface (Leighton,

T. G., 1994)'These jets and associated shock waves can cause substantial surface damage and expose fresh, highly heated surfaces. Ultrasonic irradiation of liquidpowder suspensions produces another effect: high-velocity inter-particle collisions. Cavitation and the shock waves it creates in a slurry can accelerate solid particles to high velocities (Suslick, K. S. and Doktycz, S. J., 1990). The resultant collisions are capable of inducing dramatic changes in surface morphology, composition, and reactivity (Suslick, K. S. and Doktycz, S. J., 1990).

Sonochemistry can be roughly divided into categories based on the nature of the cavitation event: homogeneous sonochemistry of liquids, heterogeneous sonochemistry of liquid-liquid or liquid-solid systems, and sonocatalysis (which overlaps the first two). In some cases, ultrasonic irradiation can increase reactivity by nearly a millionfold (Suslick, K. S. and Casadonte, D. J., 1987). Because cavitation can only occur in liquids, chemical reactions are not generally seen in the ultrasonic irradiation of solids or solid-gas systems.

Sonoluminescence in general may be considered a special case of homogeneous sonochemistry; however, recent discoveries in this field have heightened interest in the phenomenon in and by itself (Crum, L. A., 1994, S. J. Putterman, 1995). Under conditions where an isolated, single bubble undergoes cavitation, recent studies on the duration of the sonoluminescence flash suggest that a shock wave may be created within.

\section{ACOUSTIC CAVITATION}

Sonochemistry and sonoluminescence were derived principally from acoustic cavitation, which serves as an effective means of concentrating the diffuse energy of sound. Compression of a gas generates heat. When the compression of bubbles occurs during cavitation, it is more rapid than thermal transport, which generates a short-lived, localized hot-spot. Rayleigh's early descriptions of a mathematical model for the collapse of cavities in incompressible liquids predicted enormous local temperatures and pressures (Lord Rayleigh, 1917) Ten years later, Richards and Loomis reported the first chemical and biological effects of ultrasound (Richards, W. T. and Loomis, A. L., 1927) 
If the acoustic pressure amplitude of a propagating acoustic wave is relatively large (greater than $0.5 \mathrm{MPa}$ ), local in homogeneities in the liquid can give rise to the rapid growth of a nucleation site into a cavity of macroscopic dimensions, primarily filled with vapor. Such a bubble is inherently unstable, and its subsequent collapse can result in an enormous concentration of energy (Figure 1). This violent cavitation event is generally termed "transient cavitation." A normal consequence of this unstable growth and subsequent collapse is that the cavitation bubble itself is destroyed. Gasfilled remnants from the collapse, however, may give rise to reinitiation of the process.

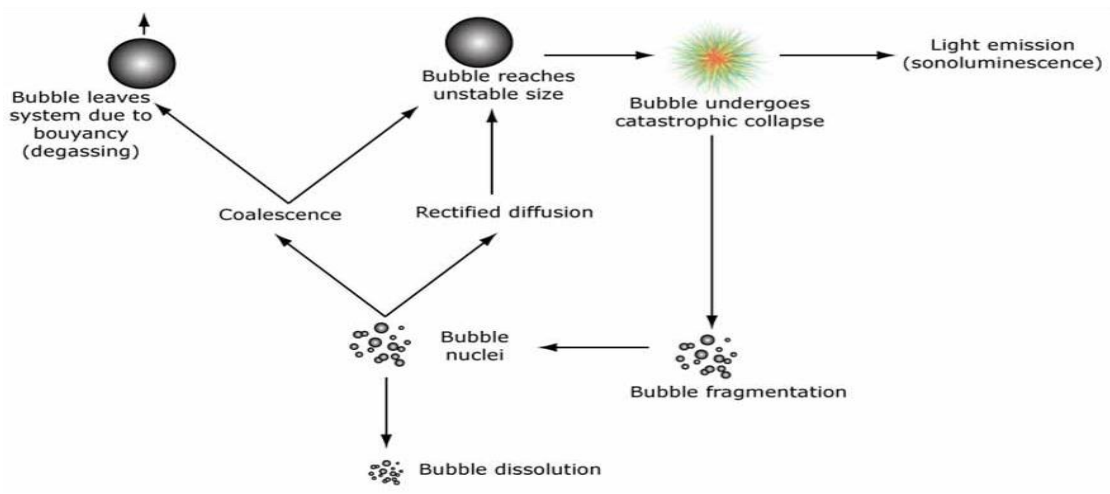

Fig. (1): Bubble nuclei under the influence of an acoustic sound field

A variety of devices have been used for ultrasonic irradiation of solutions. There are three general designs in use presently: the ultrasonic cleaning bath, the direct immersion ultrasonic horn, and flow reactors. The originating source of the ultrasound is generally a piezo ceramic material that is subjected to a high Alternating current(AC) voltage with an ultrasonic frequency (typically $15-50 \mathrm{kHz}$ ). The vibrating source is attached to the wall of a cleaning bath, to an amplifying horn, or to the outer surfaces of a flow-through tube or diaphragm. The ultrasonic cleaning bath is clearly the most accessible source of laboratory ultrasound and has been used successfully for a variety of materials. The most intense and reliable source of ultrasound generally used in the chemical laboratory is the direct immersion ultrasonic horn $(50$ to $500 \mathrm{~W} \mathrm{~cm})$, as shown in (Figure 2) which can be used for work under either inert or reactive atmospheres or at moderate pressures ( $<10 \mathrm{~atm}$.). These devices are available from several manufacturers at modest cost.

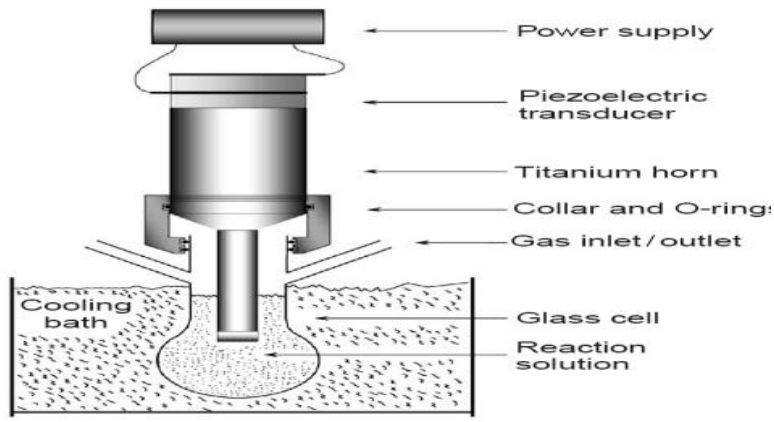

Fig. (2): A typical sonochemical apparatus with direct immersion ultrasonic horn. Ultrasound can be easily introduced into a chemical reaction with good control of temperature and ambient atmosphere 


\section{THEORY}

The generally accepted explanation for the origin of sonochemistry and sonoluminescence is the hot-spot theory, in which the potential energy given to the bubble as it expands to maximum size is concentrated into a heated gas core as the bubble implodes. The oscillations of a gas bubble driven by an acoustic field are well described by the "Rayleigh-Plesset'"equation (McNamara III, et al, 1999).

These kinetic studies revealed that there were in fact two sonochemical reaction sites: the first (and dominant site) is the bubble's interior gas phase while these condition initially liquid phase. The latter corresponds either to heating of a shell of liquid around the collapsing bubble or to droplets of liquid ejected in to the hot- spot by surface wave distortions of the collapsing bubble.

\section{Microjet Formation During Cavitation at Liquid-Solid Interfaces}

A very different phenomenon arises when cavitation occurs near extended liquid-solid interfaces. There are two proposed mechanisms for the effects of cavitation near surfaces: micro jet impact and shock wave damage. Whenever a cavitation bubble is produced near a boundary, the asymmetry of the liquid particle motion during cavity collapse induce side formation in the cavity (McNamara III, et al, 1999). The potential energy of the expanded bubble is converted into kinetic energy of a liquid jet that extends through the bubble's interior and penetrates the opposite bubble wall. Because most of the available energy is transferred to the accelerating jet, rather than the bubble wall itself, this jet can reach velocities of hundreds of meters per second. Because of the induced asymmetry, the jet often impacts the local boundary and can deposit enormous energy densities at the site of impact. Such energy concentration can result in severe damage to the boundary surface. The second mechanism of cavitation-induced surface damage invokes shock waves created by cavity collapse in the liquid. The impingement of micro jets and shock waves on the surface creates the localized erosion responsible for ultrasonic cleaning and many of the sonochemical effects on heterogeneous reactions. The erosion of metals by cavitation generates newly exposed, highly heated surfaces (Figure 3).

A solid surface several times larger than the resonance bubble size is necessary to induce distortions during bubble collapse. For ultrasound of $20 \mathrm{kHz}$, damage associated with jet formation cannot occur if the solid particles are smaller than 200 $\mathrm{mm}$.

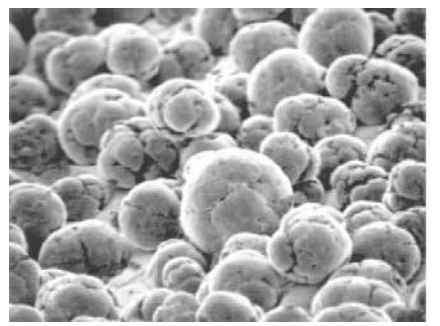

Before ultrasound $100 \mu \mathrm{m}$

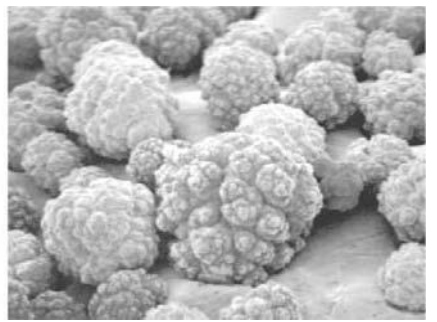

60 min. ultrasound

Fig.(3): The effect of ultrasonic irradiation on the surface morphology and particle size of Ni powder. High-velocity inter particle collisions caused by ultrasonic irradiation of slurries are responsible for the smoothing and removal of passivating oxide coating. 


\section{SONOLUMINESCENCE}

In addition to driving chemical reactions, ultrasonic irradiation of liquids can also produce light. Sonoluminescence was first observed from water by (Frenzel, H. and Schultes, H. Z., 1934) .

As with sonochemistry, sonoluminescence were derived from acoustic cavitation. It is now generally thought that there are two separate forms of sonoluminescence: multiple-bubbles sonoluminescence (MBSL) and single-bubble sonoluminescence (SBSL) (Crum, L. A., 1994, S. J. Putterman, 1995) .

Since cavitation is a nucleated process and liquids generally contain large numbers of particulates that serve as nuclei, the "cavitation field" generated by a propagating or standing acoustic wave typically consists of very large numbers of interacting bubbles, distributed over an extended region of the liquid. If this cavitation is sufficiently intense to produce sonoluminescence, then the phenomenon is MBSL (Suslick,K.S. and Crum,L.A., 1997) .

\section{MECHANISM}

Homogeneous Sonochemistry: Bond Breaking and Radical Formation

The chemical effects of ultrasound on aqueous solutions have been studied for many years. The primary products are $\mathrm{H}_{2}$ and $\mathrm{H}_{2} \mathrm{O}_{2}$; there is strong evidence for various high-energy intermediates, including $\mathrm{HO}_{2}, \mathrm{H}$ and $\mathrm{OH}$ (Riesz, P., 1991). The sonolysis of water, which produces both strong reductants, and oxidants is capable of causing secondary oxidation and reduction reactions, as often observed by (Margulis,M.A. and Maximenko,N., 1991) . Most recently there has been strong interest shown in the use of ultrasound for remediation of low levels of organic contamination of water (Destaillats,

$\mathrm{H}$., et al,2001 ). The $\mathrm{OH}$ - radicals produced from the sonolysis of water are able to attack essentially all organic compounds (including halocarbons, pesticides, and nitro aromatics) and through a series of reactions oxidize them fully. The ultrasonic irradiation of organic liquids creates the same kinds of products associated with very high temperature pyrolysis (Suslick, K. S., et al, 1983). The sonochemistry of solutes dissolved in organic liquids also remains largely unexplored. The sonochemistry of metal carbonyl compounds is an exception (Suslick, K. S., 1986). Detailed studies of these systems led to important mechanistic understandings of the nature of sonochemistry. A variety of unusual reactivity patterns have been observed during ultrasonic irradiation, including multiple ligand dissociation, novel metal cluster formation, and the initiation of homogeneous catalysis at low ambient temperature (Suslick, K. S., 1999) . hydrolysis of nitriles in acidic or basic conditions as hydrolysis of benzonitiles under thermal or ultrasound using $2 \mathrm{M} \mathrm{HCl}$ or $2 \mathrm{M} \mathrm{NaOH}$ with improvement of reaction time from 8h. to 45min.(P.Lignier,2001).(Figure4).

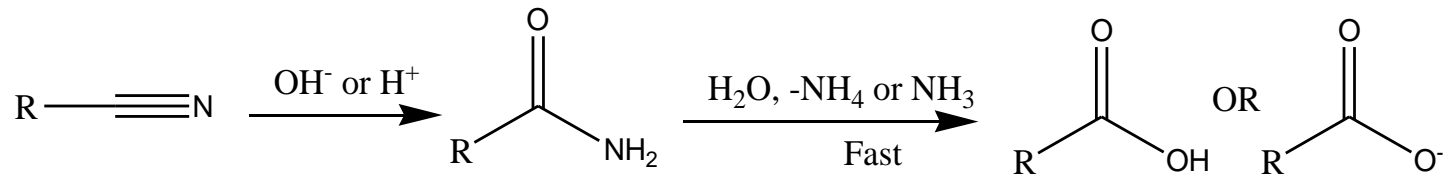

Hydrolysis of a nitrile in acidic or basic conditions 


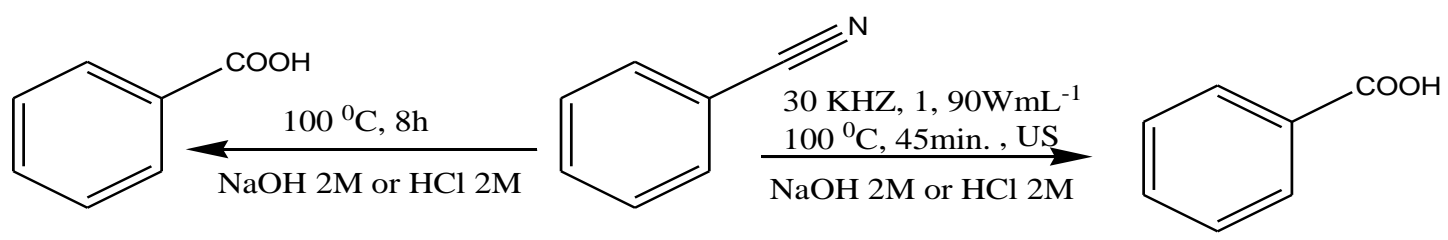

Experimental conditions for hydrolysis of benzonitrile under thermal or ultrasonic activation in acidic or basic conditions.

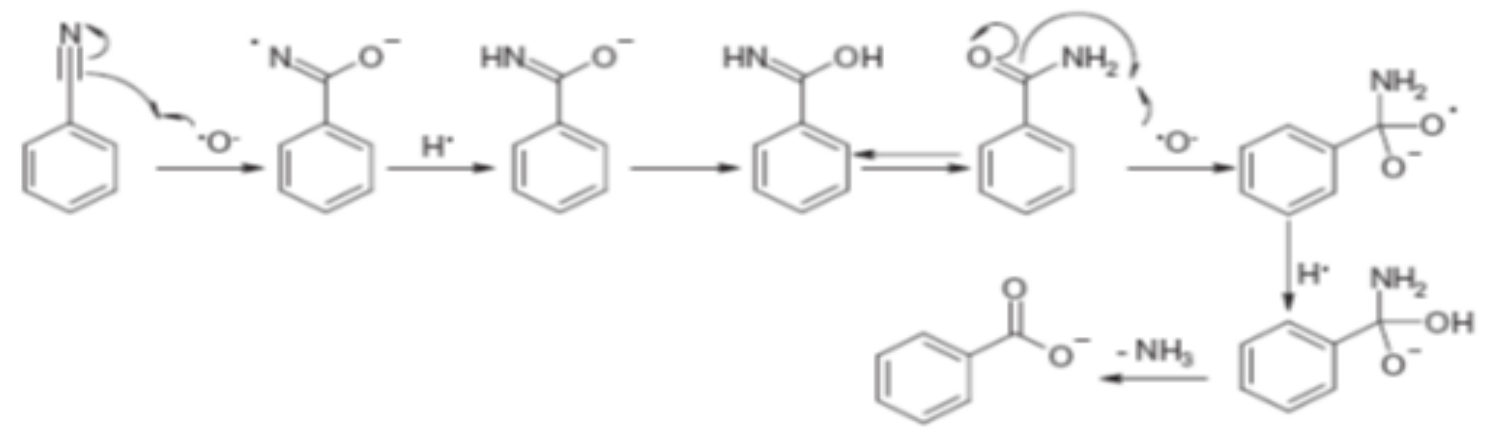

Hypothesis of the mechanism of sono-hydrolysis of nitrile in basic conditions

Fig. (4)

\section{Application of Ultrasound in Chemical Synthesis :-}

1. Transformation of aromatic and aliphatic alcohols in the equivalent carboxylic acids and ketones.

Various aromatic, aliphatic and conjugated alcohols were transformed into the corresponding carboxylic acids and ketones in good yields with aq. $70 \% \mathrm{t}-\mathrm{BuOOH}$ in the presence of catalytic amounts of bismuth(III) oxide. This method does not involve cumbersome work-up, exhibits chemoselectivity and proceeds under ambient conditions (Malik P., D. and Chakraborty D., 2010) as shown in Figure (5).

$$
\begin{aligned}
& 0.1 \text { eq. } \mathrm{Bi}_{2} \mathrm{O}_{3} \\
& \mathrm{R}^{2} \mathrm{OH} \frac{5 \text { eq. } t \mathrm{BuOOH}(70 \% \mathrm{aq})}{\mathrm{EtOAx}, \text { r.t. }, 18-55 \mathrm{~h}}=
\end{aligned}
$$

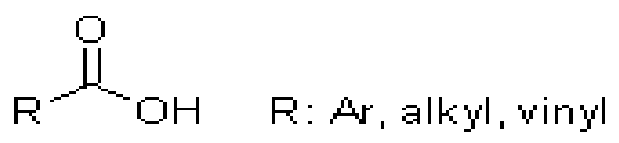

Fig. (5)

\section{Some name Reactions}

\section{a- Enantioselective Michaell addition}

A highly enantioselective Michael addition of malonates to $\alpha, \beta$-unsaturated ketones in water is catalyzed by a primary-secondary diamine catalyst containing a long alkyl chain. This asymmetric Michael addition process allows the conversion of various $\alpha, \beta$ unsaturated ketones (Z. Mao, et al, 2010) as shown in Figure (6) 
<smiles>CC(=O)C[C+](C)C(C)C</smiles>

Fig. (6)

\section{b- Aza-Michael addition of nucleophiles to $\alpha, \beta$-unsaturated compounds}

The literature has reported several instances of sonochemical solvent-free procedures in which at least one reagent/reactant is a liquid. One of these is the synthesis of $\mathrm{N}$-heterocycles via the aza-Michael addition of nucleophiles to $\alpha, \beta$ unsaturated ketones, esters, and nitriles to form carbon-nitrogen bonds as described by (Bandyopadhyay, D. et al., 2012). High yields were obtained in water (98\%) as well as in solvent-free conditions (93\%), although the former was much faster as shown in Figure (7)

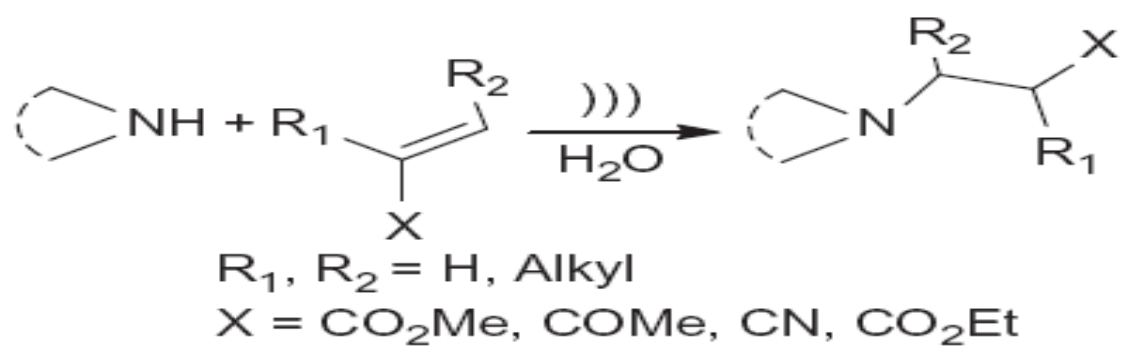

Fig. (7)

\section{c- Borono-Mannich reactions in solvent-free conditions}

Borono-Mannich reactions can be performed in solvent-free conditions under microwave irradiation with short reaction time. Full conversion of the starting materials towards the expected product was achieved, starting from stoichiometric quantities of reactants, avoiding column chromatography. No purification step other than an aqueous washing was required (P. Nun, et al, 2010) as shown in Figure (8)

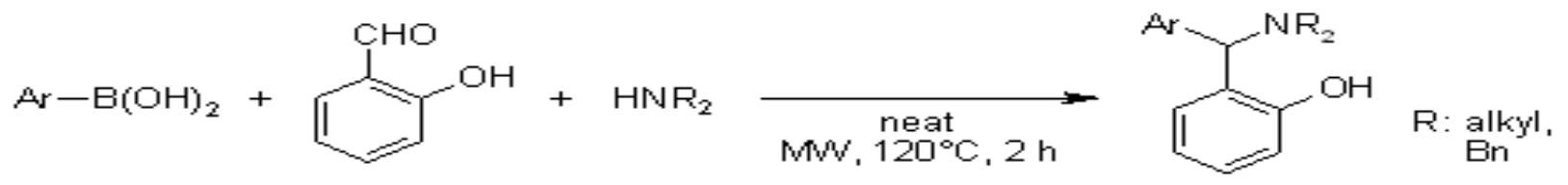

Fig. (8)

\section{d- Barbier-Grignard type reactions}

The Barbier-Grignard type reactions are usually referred as a transformation in which the organometalic reagents serve as nuclophiles to lead to carbon-carbon bond formations ( li, C.-J, 1996). As shown in Figure (9)

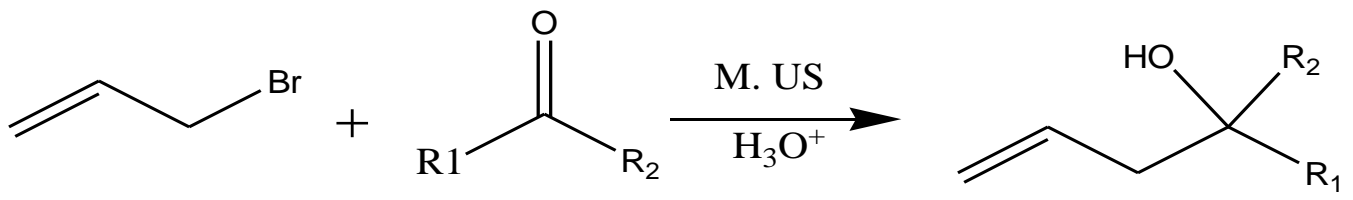

Fig. (9) 
In attemps to prepare homoallyl alcohol in an enviromental friendly process, Andrews and co-workers reported its synthesis using a solvent-free method mediated by tin ultrasound (Andrews, P.C., et al, 2002). On the other hand, carbonyl allylation reactions mediated by $\mathrm{SnCl}_{2}$ in aqueous solution without a Lewis acid under ultrasonic irradiation were successfully carried out(wang, J., et al., 2004 ), as shown in Figure (10)

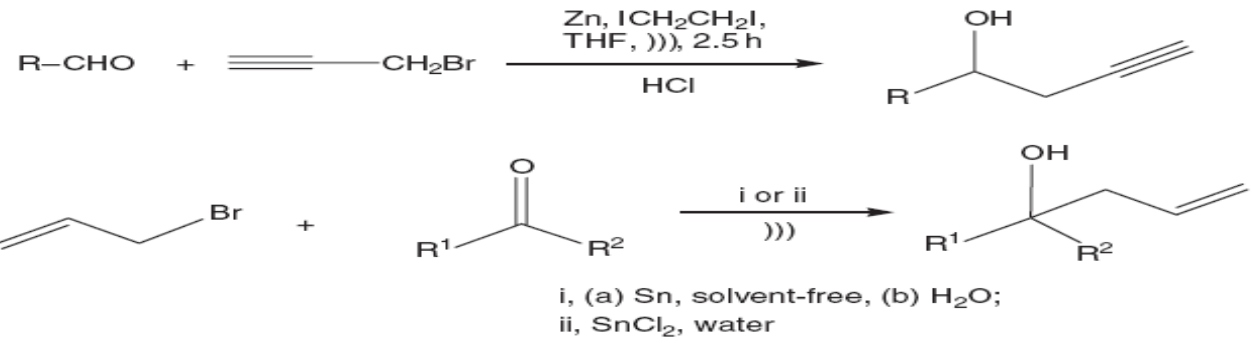

Fig. (10)

\section{e- Reformatsky reactions}

The Reformatsky reaction with aldehydes can be achieved in almost quantitative yield using either a low-intensity cleaning bath (Han, B.H. and boudjouk, P. J., 1982 ) (LIU) or a high intenisty probe sonicator(HIU) (Suslick, K. S. and doktyez, S. J., 1989), which makes this reaction one of the first organometallic reactions studied under ultrasonic irradiation because of the difficulties in metal activation under silent conditions as shown in Figure (11).

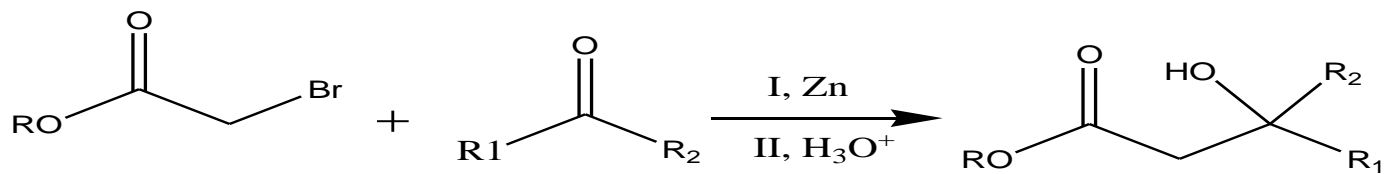

Fig. (11)

Recently, Ross and Battsch reported successful syntheses of $\beta$-hydroxyester using phenyl ketons, $\alpha$-bromoesters zinc dust, and catalytic amount of iodine under high-intenisty ultrasonic irradiation with good to quantitative yield (Ross, N. A. and Bartsch, R. A., 2003 ) as shown in Figure (12).

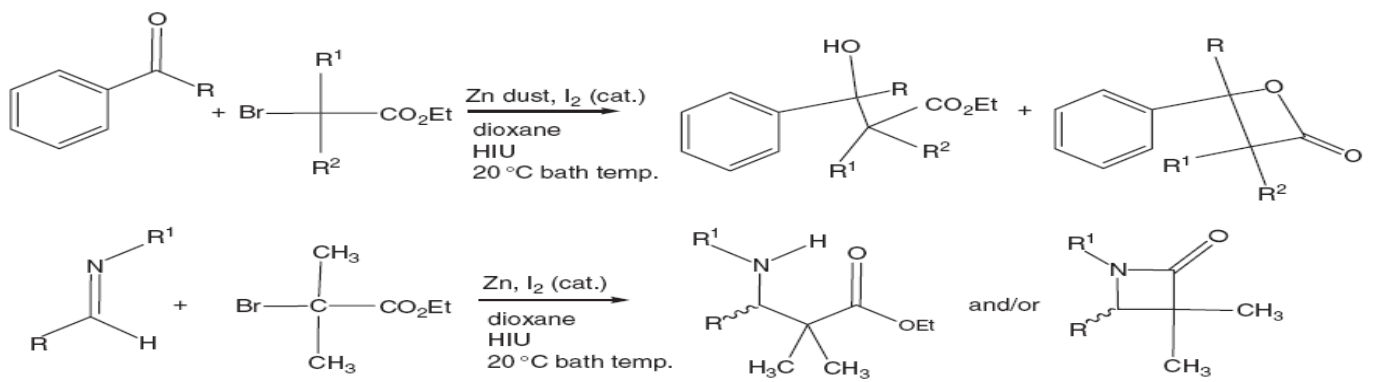

Fig. (12)

Reformatsky reaction was used by (Lindy, J., et al., 1986,). Ultrasonic radiation has been found to accelerate the Reformatsky reaction between ethyl bromoacetate and cyclopentanone generated in $30 \mathrm{~min}$ at $25^{\circ} \mathrm{C}$ compared with $80 \%$ in $12 \mathrm{~h}$ at $80^{\circ} \mathrm{C}$ by conventional methods as shown in Figure (13) 
<smiles>C=C1CCCC1</smiles>
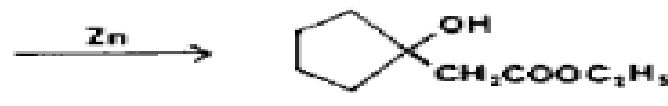

cyclopentanone

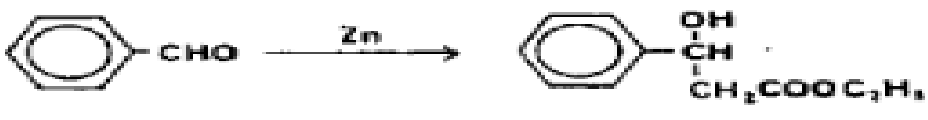

Fig. (13)

\section{f- Ulmann-type coupling reactions}

The Ulmann-type coupling reaction occurs at a lower temperature and shorter time in almost quantitative yield with probe sonication (Lindy, J., et al., 1986, Citas, P. and Luche, J.L., 1998) as shown in Figure (14).

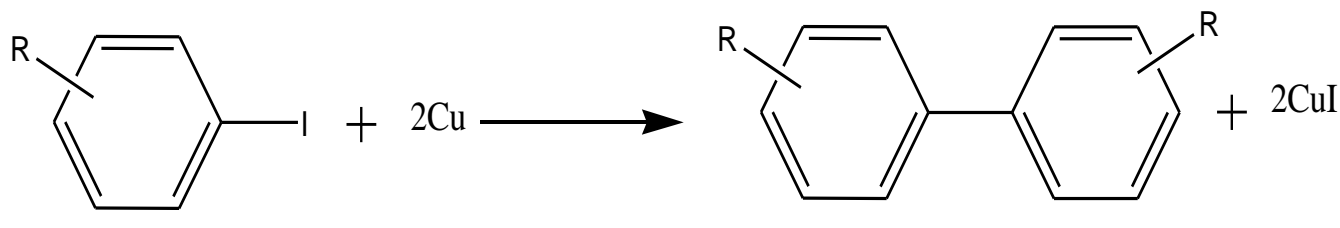

Fig.

(14)

Having observed the improvement using ultrasound in the cross-coupling of $\mathrm{O}$ halobenzoic acids and substituted benzoamines with copper or $\mathrm{Cu} / \mathrm{Zn}$ under probe sonication (Hanoun, J.P, et al, 1995). Robin and co-workers successfully synthesized acridine derivatives using this method (Robin, M., et al, 2002). In order to replace dry organic solution with more environmentally benign ones: Pellon et al. extended the synthesis of halobenzoic acid and alkylamine in the presence of water as a solvent with copper under probe sonication (Pellon, R. F., et al, 2005 ). In most cases the yields were $20 \%$ greater than those resulting from classic heating.

(Lindy, J., et al., 1986,) found that the Ulmann coupling of arylhalides to yield biaryls is widly used in aromatic chemistry and is normaly carried out by heating the halide (with or without solvent) in the presence of copper powder as shown in Fig. (15)
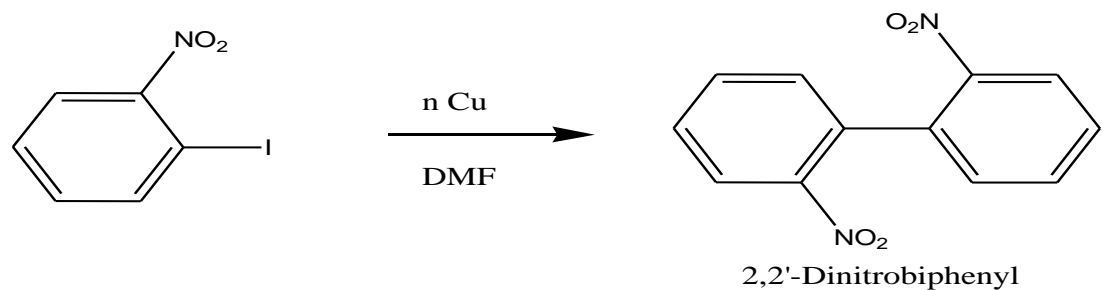

Fig.(15)

\section{g- Simmons-Smith reaction}

Sonochemically activated metals greatly improved the reaction of $\mathrm{CH}_{2} \mathrm{X}_{2}(\mathrm{X}=\mathrm{Br}$, I) with olefin to produce cyclopropane derivatives as shown in Fig. (16)<smiles>[R]C([R])=C([2H])[10BH2]</smiles>

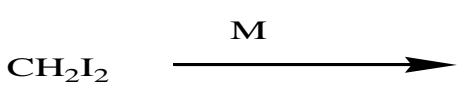<smiles>[2H]C1([10BH2])CC1([2H])[13CH3]</smiles> 
Fig. (16)

The reaction condition of cyclopropanation of enoxysilone $\left(\mathrm{R}_{1} \cdot \mathrm{R}_{2}=\mathrm{H}: \mathrm{R}_{3}=\mathrm{Ph}: \mathrm{R}_{4}=\mathrm{OSiM}{ }_{3}\right)$ with dihalomethane were studied by (Sun, H,-Z. and Li, J.S., 1998) ${ }^{(45)}$. Zhu carried out the cycloaddition of $\mathrm{C}_{60}$ and methanofullerene dihalides, $\mathrm{C}_{60}\left(\mathrm{CX}_{2}\right)(\mathrm{X}=\mathrm{Cl}, \mathrm{BR}, \mathrm{I})$, with $\mathrm{Mg}$ in the ionic liquid 1-butyl-3methylimidazolium tetrafluroborate $[\mathrm{BMIM}] \mathrm{BF}_{4}$ with a yield of $55-84 \%$, in contrast to 6-21\% yield in THF under sonication (Zhu, Y , 2004) ${ }^{(46)}$ as shown in Fig. (17)

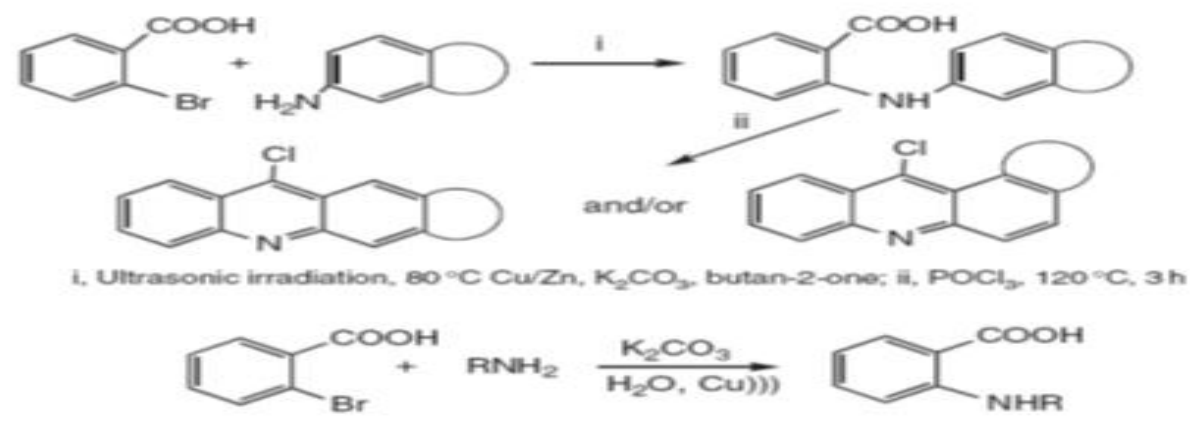

\section{h- Suzuki Coupling reactions}

Fig. (17)

Suzuki reaction, especially those friendly to the environment, that involve ultrasonic irradiation have been reviewed recently (Bai, L. and Wang, J.X., 2005). Cravotto and co-workers carried out ligand-free $\mathrm{Pd} / \mathrm{C}$ catalyzed Suzuki cross- and homo-coupling in either water or DMF using high-intensity ultrasound (Cravotto, G., et $a l$, 2005). In combination with microwave (MW) irradiation, further improvements in yields and reaction time were achieved. The authors also reported that Ulmann homocoupling mediated by zinc also occurred, with $\mathrm{CO}_{2}$ bubbling into the reaction. The failure of 3-arylation of 3-bromo-4-hydroxycoumarin using the Suzuki procedure under oxygen atmosphere irradiated by ultrasound was investigated (Cravotto, G., et al, 2005), and a mechanism was proposed.

Besides the study on Suzuki cross-coupling reaction in the ionic 1,3-di-n-butylimidazolium tetrafluoroborate $\left.9[\mathrm{BBIM}]] \mathrm{BF}_{4}\right]$ ) catalyzed by palladium prepared in situ in a cleaning bath (Rajagopal, R., et al, 2002). Deshmukh and co-workers reported ligand-free Pd-catalyzed coupling between aryl halides and terminal alkyne (Deshmukh, R.R., et al, 2001 ) as shown in Figure (18)

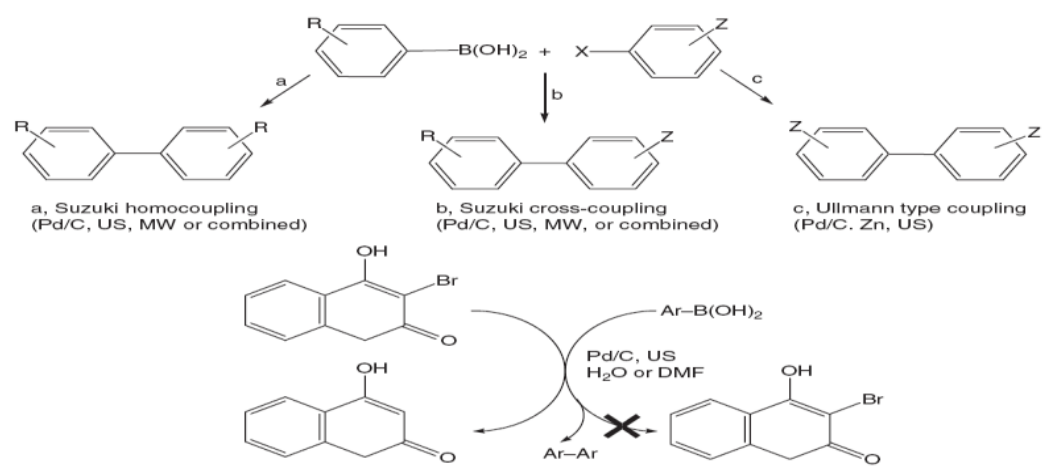

Fig. (18) 


\section{i- Heck reaction}

Sonogashira reaction or terminal acetylenes (Gholap, A.R., et al, 2005) (Heck reaction) in ionic liquids using a cleaning bath. (Ambulgekur, G. V., et al, 2005) investigated the leaching of Pd off support during the reaction, by examining the Heck reaction under ultrasound. Besides noticing that the reaction was accelerated by ultrasound, the authors reported that the $\mathrm{Pd} / \mathrm{C}$ catalyst can be reduced by sodium formate and redeposited, so that the catalyst can be recycled twice as shown in Figure (19).

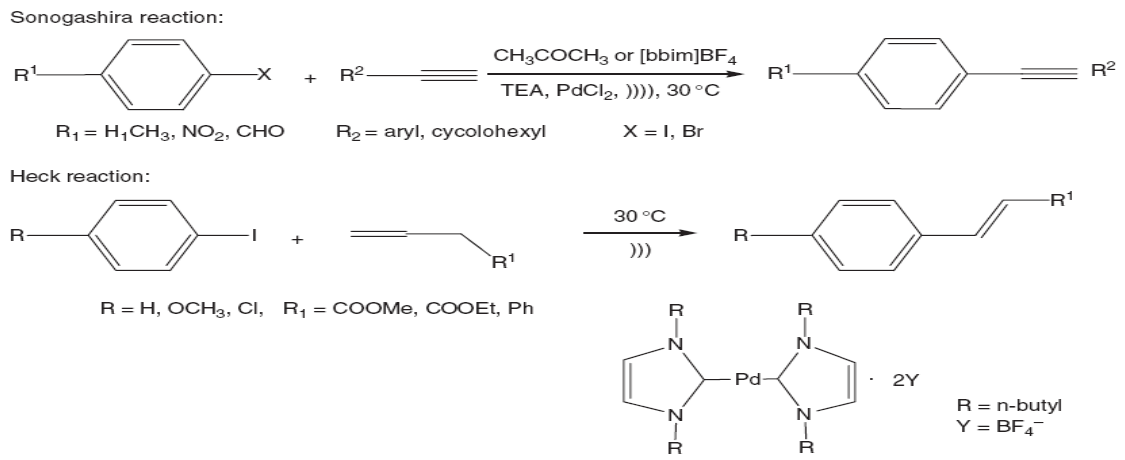

Fig. (19)

\section{j- Diels-Alder cycloadditions}

(Bravo, J. L., et al, 2006) have shown that Diels-Alder cycloadditions, conducted at relatively low temperatures, can be efficiently activated by ultrasound using an ionic liquid (IL) as the reaction medium as shown in Figure (20)

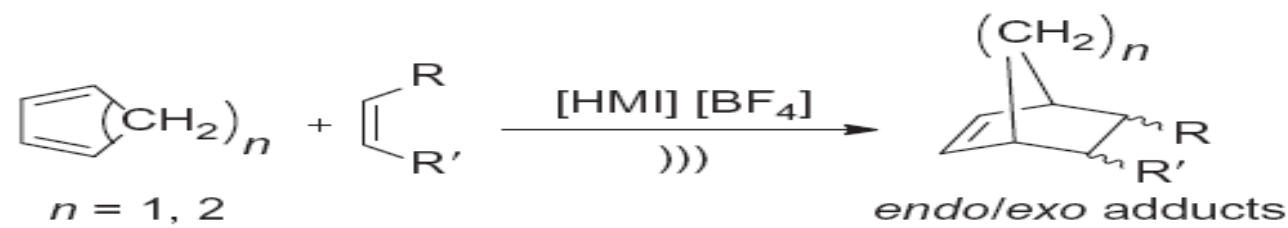

Fig. (20)

3. Organocatalytic direct $\alpha$-hydroxy phosphonate of aldehydes and ketones

An organocatalytic, direct synthesis of $\alpha$-hydroxy phosphonates via reaction of aldehydes and ketones with trimethylphosphite in the presence of catalytic amounts of pyridine 2,6-dicarboxylic acid in water is simple, cost-effective and environmentally benign (F. Jahani, et al, 2010) as shown in Figure (21).

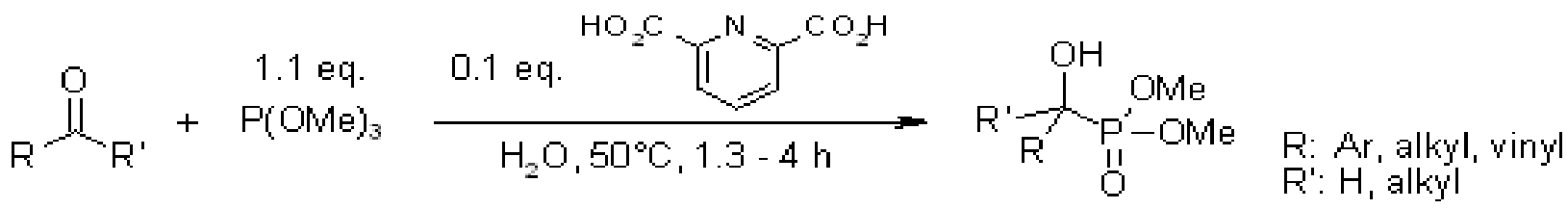

Fig. (21)

\section{Intermolecular addition of perfluoroalkyl radicals}

Intermolecular addition of perfluoroalkyl radicals on electron rich alkenes and alkenes with electron withdrawing groups in water, mediated by silyl radicals gives perfluoroalkyl-substituted compounds in good yields. The radical triggering events employed consist of thermal decomposition of 1,1'- azobis(cyclohexanecarbonitrile) 
(ACCN) or dioxygen initiation (S. Barata, et al, 2010) as shown in Figure (22)

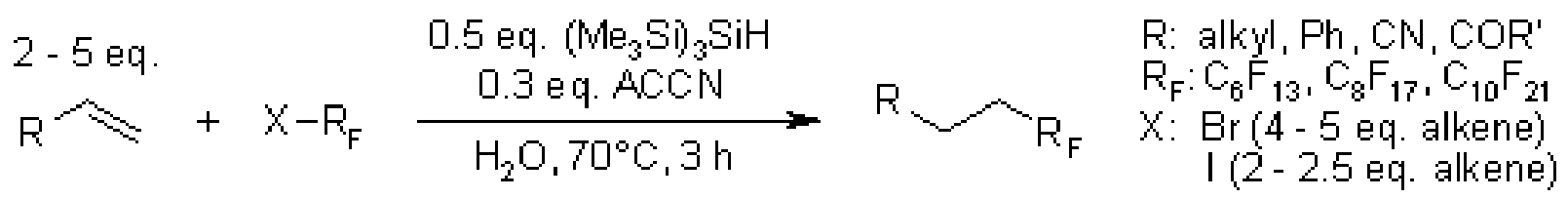

Fig. (22)

\section{N-formylation.}

\section{a. Practical catalytic method for $N$-formylation}

A simple, practical, and catalytic method for the $N$-formylation in the presence of molecular iodine as a catalyst under solvent-free conditions is applicable to a wide variety of amines. $\alpha$-Amino acid esters can be converted without epimerization (J. G. Kim, and D. O. Jang, 2010) as shown in Figure (23).

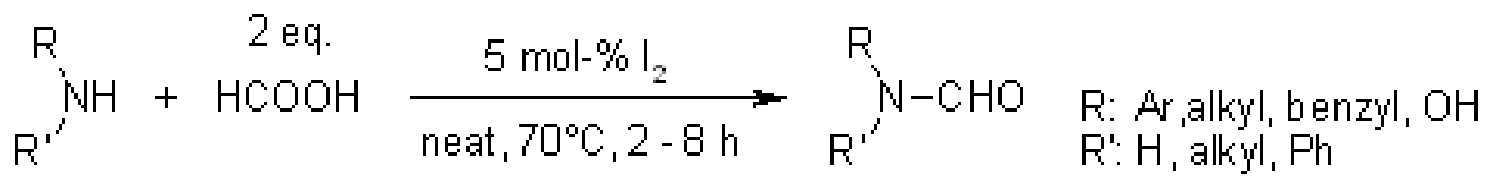

Fig.(23)

\section{b. Mild method for $N$-formylation in the presence of Indium metal}

A simple, mild method for $N$-formylation in the presence of indium metal as a catalyst under solvent-free conditions is applicable to the chemoselective reaction of amines and $\alpha$-amino acid esters without epimerization (J. G. Kim, and D. O. Jang, 2010) as shown in Figure (24).

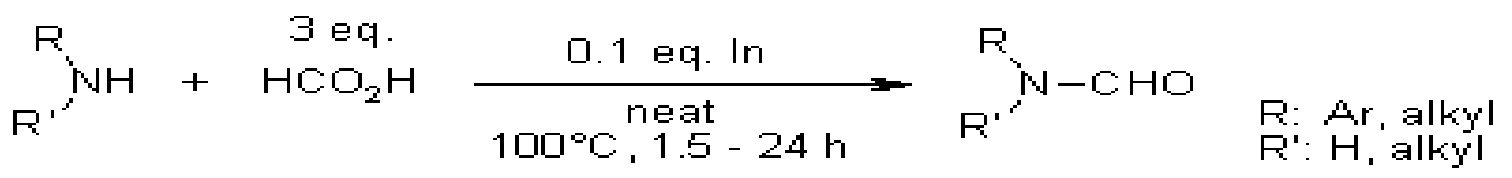

Fig.(24)

\section{Direct oxidation of methyl group in aromatic nucleus}

A methyl group at an aromatic nucleus is oxidized directly to the corresponding carboxylic acid in the presence of molecular oxygen and catalytic hydrobromic acid under photoirradiation (S. I. Hirashima, and A. Itoh, 2006) as shown in Fig. (25)

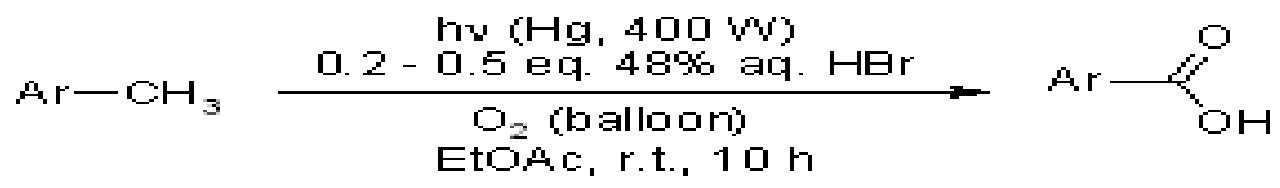

Fig. (25)

\section{Oxidation of sulfides with $\mathrm{H}_{2} \mathrm{O}_{2}$}

Oxidation of sulfides with $30 \%$ hydrogen peroxide catalyzed by tantalum carbide provides the corresponding sulfoxides in high yields, whereas niobium carbide as catalyst efficiently affords the corresponding sulfones. Both catalysts can easily be 
recovered and reused without losing their activity (M. Kirihara, et al, 2010) as shown in Figure (26).

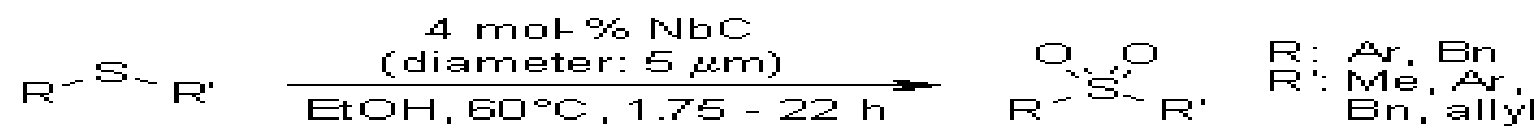

Fig. (26)

\section{Oxidation of alkynes in aqueous media}

Oxidation of alkynes using ammonium persulfate and diphenyl diselenide as catalyst in aqueous media leads to 1,2-unprotected dicarbonyl derivatives or to hemiacetals starting from terminal alkynes (S. Santoro, et al, 2010) as shown in Figure (27).

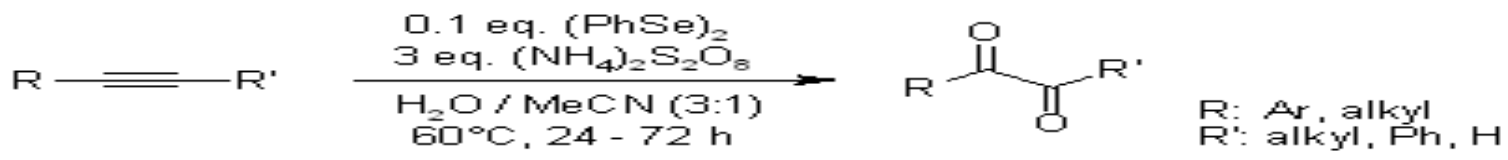

Figure (27)

\section{REFERENCE}

Ambulgekur, G. V.; Bhange, B. M.; Damant. S. D. . (2005) Tetrahedron Let, 46, 2483-2485.

Andrews, P.C.; peat, A. C.; ( 2002) raston, C.L. tetrahedron let., 43, 7541-7543.

Bai, L.; Wang, J.-X.curr. (2005) Org. Chem., 9, 535-553.

Bandyopadhyay, D., Mukherjee, S., Turrubiartes, L.C., Banik, B.K., (2012). Ultrasound-assisted aza-Michael reaction in water: a green procedure. Ultrason. Sonochem. 19, 969-972.

Bravo, J.L.,Lo`pez,I., Cintas,P., Silvero,G.,Are’valo, M.J., (2006), Sonochemical cycloadditions inionic liquids. Lessons from model cases involving common dienes and carbonyl dienophiles. Ultrason. Sonochem. 13, 408-414.

Citas, P.: Luche, J.-L. (1998) in Synthetic Organic Sonochemistry, Luche, J.- L., Plenum: New York, ; pp167-234

Cravotto, G.; Beggiato, m.; Penoni,A.; Palmisano, g.; Tollari, S.; Levenque, (2005) J.-L.; Bonrath, W. Tetrahedron Len., 46, 2267-2271.

Cravotto, G.; Palmisano,G.; Tollari, S.; Nano, G. M.; (2005) Penoni, A. Ultrason. Sono Chom., 12, 91-91.

Crum, L. A. , (1994) Phys. Today, 47, 22-29.

Deshmukh, R.R.; Rajagopal, R.; Srinivasan, K. (2001) Chem. Commun., 15441545 .

Destaillats, H.; Lesko, T. M.; Knowlton, M.; Wallace, H.; Hoffmann, M. R. .(2001) Ind. Eng. Chem. Res, 40, 3855-3860.

D.J. Brown, in: A. Katritzky, C.W.(1984) Rees (Eds.), Comprehensive Heterocyclic Chemistry, vol. 13, Pergamon Press, Oxford, .

F. Jahani, B. Zamenian, S. Khaksar, M. (2010) Taibakhsh, Synthesis, , 3315-3318. 
Flint, E. B.; Suslick, (1991) K. S. Science, 253, 1397-1399.

Frenzel, H.; Schultes, . (1934) H. Z. Phys. Chem, 27b, 421-424.

Gholap, A.R.; Venkatesan, K.; Pasricha, R.; Daniel, T.; Lahoti, (2005) R. J.; Srinivasan, K.V. J. Org. Chem., 70, 4869-4872.

Han, B.H.; boudjouk, (1982) P. J.j org. chem., 47, 5030-5032.

Hanoun, J.-P.;Galy, (1995) J. P.; Tenaglia, A. Synth. Commun., 25, 2443-2448.

H. Xu, W.M. Liao, H.F. Li, (2007) A mild and efficient ultrasound-assisted synthesis of diaryl ethers without any catalyst, Ultrason. Sonochem. 14, 779-782.

J. G.Kima, D.O. (2010) Jang, Syn lett, Reaction of amines and alpha- amino acid esters without epimerization. , 1231-1234.

J. G. Kim, D. O. Jang,( 2010) Syn lett, , 2093-2096.

K. Jadidi, R. Gharemanzadeh, M. Mehrdad, H.R. Darabi, H.R. Khavasi, D. Asgari, (2008) A facilesynthesis of novel pyrrolizidines under classical and ultrasonic conditions, Ultrason. Sonochem. 15, 124-128.

K.P. Guzen, A.S. Guarezemini, A.T.G. Orfao, R. Cella, C.P. Pereiraa, (2007) H.A. Stefani, Eco-friendly synthesis of imines by ultrasound irradiation, Tett. Lett. 48,1845-1848 .

Leighton, T. G. (1994), The Acoustic Bubble Academic Press: London.

li, (1996) C.-J. tetrahedron, 52, 5643-5668.

Lindy, J.;lorimer, J. P.; Mason, (1986) T. J. Ultrasonics, 24, 292-293.

Lord Rayleigh,( 1917) Philos. Mag. Ser. 6, 34, 94-98.

Malik P., D. Chakraborty D. Bismuth(2010) (III) oxide catalyzed oxidation of alcohols with tert-butyl hydroperoxide, Synthesis, 3736-3740.

Margulis,M.A.(1991),Maximenko,N.A.Adv.Sonochem2,253-292.

McNamara III, W. B.; Didenko, Y.; Suslick, (1999) K. S. Nature, 401, 772-775.

M. Kirihara, A. Itou, T. Noguchi, (2010), J. Yamamoto, Synlett, 1557-1561.

Pellon, R. F.; Estevez-Braun, A. Docampo, M. L. Martin ,A.; Ravelo, . (2005) A. G. Syn lett, 1606-1608.

P.Lignier(2011) Ultrasonics Sonochemistry 18, 28-31.

P. Nun, J. Martinez, F. Lamaty(2010), Synthesis, 2063-2068.

Rajagopal, R.; Jaricote, . (2002), D. V.; Srinivasan, K. V. Chem. Commun616-617.

Richards, W. T.; Loomis, (1927)A. L. J. Am. Chem. Soc., 49, 3086-3100.

Riesz, (1991) P. Adv. Sonochem., 2, 23-64.

Robin, M.; Pique, V.; Faure, R.; Galy, (2002) J.-P. J. Heterocycl.Chem., 39, 10831085.

Ross, N. A.; Bartsch, (2003) R. A J. Org. Chem., 68, 360- 366.

S. Barata-Vallejo, A. Postigo, (2010) J. Org. Chem., , 75, 6141-6148.

S. I. Hirashima, A. Itoh, (2006) Synthesis, , 1757-1759.

S. J. (1995) Putterman, Sci. Am., (Feb.), 46-52.

S. Santoro, B. Battistelli, B. Gjoka, (2010) C.-w. S. Si, L. Testaferri, M. Tiecco, C. Santi, Synlett, , 1402- 1406.

Sun, H,-Z.; Li, (1998) J.-S. Chin. J. Org. Chem., 18, 550- 556.

Suslick, K. S. (1986) Adv. Organomet. Chem., 25, 73-119.

Suslick, K. S.; (1987) Casadonte, D. J. J. Am. Chem. Soc., 109, 3459-3461. 
Suslick, K. S.; Cline, Jr., R. E.; (1986), Hammerton, D. A. J. Am. Chem. Soc. 108, 5641-5642.

Suslick,K.S.;Crum,L.A.,(1997); Sonochemistry and Sonoluminescence. In Encyclopediaof Acoustics ; Crocker,M J.,Ed.,Wiley-Interscience NewYork Vol.1,pp271 -282.

Suslick, K. S.; (1990) Doktycz, S. J. Adv. Sonochem., 1, 197-230.

Suslick, K. S.; (1989) Doktyez, S. J. j am. Che. Soc., 111, 2342-2344

Suslick, K. S.; (1990) Doktycz, S. J. Science, 247, 1067-1069.

Suslick, K. S.; Gawienowski, J. W.; Schubert, P. F.; (1983) Wang, H. H. J. Phys. Chem., 87, 2299-2301.

Suslick, K. S.; (1999 )Price, G. Annu. Rev. Mater. Sci., 29, 295-326.

T.J. Mason, D. (2002) Peters, Practical Sonochemistry, second ed., Ellis Horwood, London, .

T.J.Mason, (2007) Sonochemistry and the environment - Providing a "green" link between chemistry, physics and engineering, Ultrason. Sonochem. 14,476483.

wang, J.; yuan, G.; (2004) dong, C.;Q. chem. Let., , 33, 286-287.

Zhu, Y. J. (2004) Phys. Chem. Solid., 65, 349-353.

Z. Mao, Y. Jia, W. Li, R. (2010) Wang, J. Org. Chem., 75, 7428-7430.

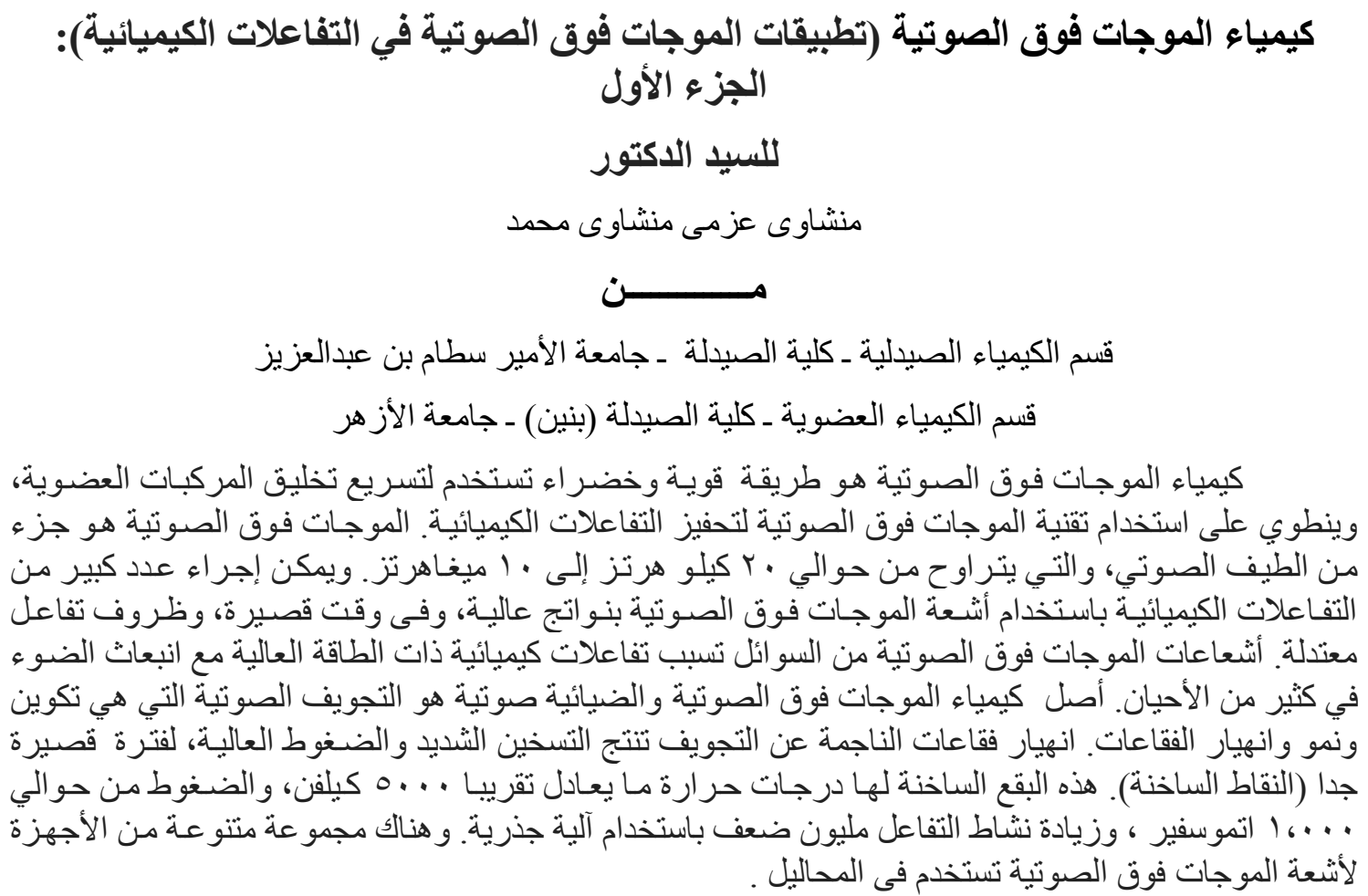

